The Astrophysical Journal Supplement Series, 154:396-401, 2004 September

(C) 2004. The American Astronomical Society. All rights reserved. Printed in U.S.A.

\title{
A "STARLESS" CORE THAT ISN'T: DETECTION OF A SOURCE IN THE L1014 DENSE CORE WITH THE SPITZER SPACE TELESCOPE
}

\author{
Chadwick H. Young, ${ }^{1}$ Jes K. Jørgensen, ${ }^{2}$ Yancy L. Shirley, ${ }^{3}$ Jens Kauffmann, ${ }^{4}$ Tracy Huard, ${ }^{5}$ Shih-Ping Lai, ${ }^{6}$ Chang Won Lee, ${ }^{7}$ \\ Antonio Crapsi, ${ }^{5}$ Tyler L. Bourke, ${ }^{8}$ Cornelis P. Dullemond, ${ }^{9}$ Timothy Y. Brooke, ${ }^{10}$ Alicia Porras,${ }^{5}$ William Spiesman, ${ }^{1}$ \\ Lori E. Allen, ${ }^{5}$ Geofrrey A. Blake, ${ }^{11}$ Neal J. Evans II, ${ }^{1}$ Paul M. Harvey, ${ }^{1}$ David W. Koerner, ${ }^{12}$ Lee G. Mundy, \\ Phillip C. Myers, ${ }^{5}$ Deborah L. Padgett ${ }^{13}$ Anneila I. Sargent, ${ }^{10}$ Karl R. Stapelfeldt, ${ }^{14}$ Ewine F. van Dishoeck, ${ }^{2}$ \\ Frank Bertoldi, ${ }^{4}$ Nicholas Chapman, ${ }^{6}$ Lucas Cieza, ${ }^{1}$ Christopher H. DeVries, ${ }^{5}$ Naomi A. Ridge, ${ }^{5}$ and Zahed Wahhaj ${ }^{12}$ \\ Received 2004 March 26; accepted 2004 April 24
}

\begin{abstract}
We present observations of L1014, a dense core in the Cygnus region previously thought to be starless, but data from the Spitzer Space Telescope show the presence of an embedded source. We propose a model for this source that includes a cold core, heated by the interstellar radiation field, and a low-luminosity internal source. The low luminosity of the internal source suggests a substellar object. If L1014 is representative, other "starless" cores may turn out to harbor central sources.
\end{abstract}

Subject headings: infrared: stars — ISM: individual (L1014) — stars: formation — stars: low-mass, brown dwarfs

\section{INTRODUCTION}

Modern astronomers first recognized dark nebulae as regions of star formation in the late nineteenth century. Molecular-line studies (e.g., Myers \& Benson 1983) showed that these dark nebulae contained copious amounts of cold material. Of these molecular cores, some were detected by the Infrared Astronomical Satellite (IRAS) and others, so-called starless cores, were not. With the Infrared Space Observatory (ISO), Ward-Thompson et al. (2002) observed 18 starless cores at 90,170 , and $200 \mu \mathrm{m}$. While they mapped the emission at 170 and $200 \mu \mathrm{m}$, only one core was detected at $90 \mu \mathrm{m}$, consistent with the idea that most of these starless cores have no associated star more luminous than $\sim 0.1 L_{\odot}$. The Spitzer Space Telescope (Werner et al. 2004) offers unprecedented sensitivity and resolution at wavelengths relevant to understanding the emission from luminous objects in dense cores. The Cores to Disks Legacy team (c2d; Evans et al. 2003) will observe over

\footnotetext{
${ }^{1}$ University of Texas at Austin, 1 University Station, C-1400, Austin, TX 78712-0259.

${ }^{2}$ Leiden Observatory, P.O. Box 9513, 2300 RA Leiden, Netherlands.

${ }^{3}$ National Radio Astronomical Observatory, P.O. Box O, Socorro, NM 87801 .

${ }^{4}$ Max-Planck-Institut Für Radioastronomie, Bonn, Germany.

5 Harvard-Smithsonian Center for Astrophysics, 60 Garden Street, MS 42, Cambridge, MA 02138.

${ }^{6}$ Astronomy Department, University of Maryland, College Park, MD 20742.

${ }^{7}$ Korea Astronomy Observatory, 61-1 Hwaam-dong Yusung-gu, Taejon 305-348, Korea.

${ }^{8}$ Harvard-Smithsonian Center for Astrophysics, Submillimeter Array Project, 645 North A'ohoku Place, Hilo, HI 96720.

9 Max-Planck-Institute für Astrophysik, Postfach 1317, D-85741 Garching, Germany.

${ }^{10}$ Division of Physics, Mathematics, and Astronomy, MC 105-24, California Institute of Technology, Pasadena, CA 91125.

${ }^{11}$ Division of Geological and Planetary Sciences, MC 150-21, California Institute of Technology, Pasadena, CA 91125.

12 Department of Physics and Astronomy, Northern Arizona University, Box 6010, Flagstaff, AZ 86011-6010.

${ }^{13}$ Spitzer Science Center, MC 220-6, California Institute of Technology, Pasadena, CA 91125.

14 Jet Propulsion Laboratory, MC 183-900, California Institute of Technology, 4800 Oak Grove Drive, Pasadena, CA 91109.
}

$15 \mathrm{deg}^{2}$ of large molecular clouds and over 120 star-forming cores, of which about 75 are considered to be starless. The only starless core observed so far is L1014, which presented a surprise. Spitzer's observations reveal an infrared point source in this supposedly "starless" core.

\section{ENVIRONMENT OF L1014}

L1014 is a Lynds opacity Class 6 dark cloud (Lynds 1962) that was undetected by both IRAS and the Midcourse Space Experiment (MSX). Visser et al. (2002) observed this core with SCUBA and concluded that L1014 is a starless core based on two criteria: (1) it was not detected by IRAS, and (2) they could not find evidence for an outflow.

While most authors have assumed a distance of $200 \mathrm{pc}$ to L1014, there is no firm evidence for this distance. The lack of foreground stars indicates that it must be closer than $1 \mathrm{kpc}$. Because it lies in the plane $\left(b=-0^{\circ} .25\right)$ near the Cygnus arm, distance estimates are unreliable. We adopt a distance of $200 \mathrm{pc}$ for L1014, but we discuss the consequences of larger distances.

\section{OBSERVATIONS AND RESULTS}

We present several new observations of L1014. First, we used the Spitzer Infrared Array Camera (IRAC) and Multiband Imaging Photometer for Spitzer (MIPS) to observe this core at wavelengths from 3.6 to $70 \mu \mathrm{m}$ (Fazio et al. 2004; Rieke et al. 2004). We also present new data from MAMBO on the IRAM $30 \mathrm{~m}$ telescope and briefly discuss new molecular-line observations with the FCRAO and the Seoul Radio Astronomy Observatory (SRAO). Finally, we include data from the Two Micron All Sky Survey (2MASS) and submillimeter observations from Visser et al. (2002).

The core of L1014 was observed with Spitzer on 2003 December 2 in all four IRAC bands (Program ID [PID] 139, Spitzer AOR key 0005163776). The observations in each band consisted of two $30 \mathrm{~s}$ images, dithered by $\sim 10^{\prime \prime}$, resulting in a generally uniform sensitivity across the $5^{\prime} \times 5^{\prime}$ field. Using a modified version of DOPHOT (Schechter et al. 1993), we identified over 2900 different sources in the IRAC images, all but one of which are consistent with being reddened background stars. 
TABLE 1

Photometry of L1014-IRS

\begin{tabular}{|c|c|c|c|}
\hline $\begin{array}{c}\lambda \\
(\mu \mathrm{m})\end{array}$ & $\begin{array}{l}S_{\nu}(\lambda) \\
(\mathrm{mJy})\end{array}$ & $\begin{array}{c}\sigma \\
(\mathrm{mJy})\end{array}$ & $\begin{array}{c}\theta_{\mathrm{ap}} \\
(\operatorname{arcsec})\end{array}$ \\
\hline $2.17 \ldots \ldots \ldots$ & 1.3 & 0.2 & 8 \\
\hline $3.6 \ldots \ldots \ldots \ldots$ & 3.6 & 0.7 & $1.7^{\mathrm{a}}$ \\
\hline ................ & 10.7 & 2.1 & $1.5^{\mathrm{a}}$ \\
\hline $5.8 \ldots \ldots \ldots \ldots$ & 17.8 & 3.6 & $1.7^{\mathrm{a}}$ \\
\hline $8.0 \ldots \ldots \ldots \ldots \ldots$ & 20.6 & 4.1 & $2.0^{\mathrm{a}}$ \\
\hline 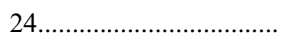 & 81.8 & 16.0 & $5.7^{\mathrm{a}}$ \\
\hline 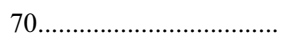 & 390 & 40 & $17^{\mathrm{a}}$ \\
\hline 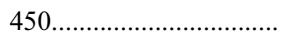 & 21500 & 16100 & 120 \\
\hline 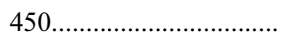 & 1070 & 230 & Peak $^{\mathrm{b}}$ \\
\hline 850 & 1800 & 400 & 120 \\
\hline 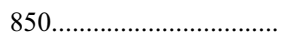 & 160 & 30 & Peak $^{\mathrm{b}}$ \\
\hline 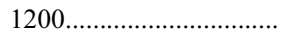 & 630 & 126 & 120 \\
\hline 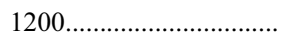 & 23 & 5 & Peak $^{\mathrm{b}}$ \\
\hline
\end{tabular}

Note.-Source at $\alpha=21^{\mathrm{h}} 24^{\mathrm{m}} 07.51, \delta=+49^{\circ} 59^{\prime} 09^{\prime \prime} 0$ (J2000.0).

${ }^{\text {a }}$ FWHM of the Spitzer point-spread profile.

${ }^{\mathrm{b}}$ In mJy beam ${ }^{-1}$.

This one source was distinct from the other objects by its IRAC colors $([3.6]-[4.5]=1.65,[5.8]-[8.0]=1.65)$; see Allen et al. (2004) and Whitney et al. (2003) for a more detailed discussion of IRAC colors. The observed fluxes were obtained by fitting interpolated, real point-spread functions to the detected sources. IRAC fluxes for the one source that is not consistent with being a reddened background star, SSTc2d $2124075+495909$, at $\alpha=21^{\mathrm{h}} 24^{\mathrm{m}} 07.51, \quad \delta=+49^{\circ} 59^{\prime} 09^{\prime \prime} 0$ (J2000.0), are listed in Table 1. We refer to this source as L1014-IRS and give all positions relative to these coordinates.

MIPS 24 and $70 \mu \mathrm{m}$ large-field photometry of L1014-IRS was done on 2003 December 10 (PID 139, Spitzer AOR key 0005164032). The 24 and $70 \mu \mathrm{m}$ integration times were 36 and $100 \mathrm{~s}$, respectively. The sky offset position was $300^{\prime \prime}$ in the scan direction (P.A. $\sim 45^{\circ}$ ). The $24 \mu \mathrm{m}$ source is shifted from the IRAC position for L1014-IRS by $(\Delta$ R.A., $\Delta$ decl. $)=$ $(-0.5,0.7)$. The $70 \mu \mathrm{m}$ source is shifted by $\left(1{ }^{\prime \prime} .8,1^{\prime \prime}\right)$. The fluxes at 24 and $70 \mu \mathrm{m}$ are in Table 1, listed with $20 \%$ uncertainty based on the calibration uncertainty as given by the Spitzer Science Center. We show a three-color image, including the 3.6, 8.0, and $24 \mu \mathrm{m}$ emission, in Figure 1 (Plate 1). L1014-IRS stands out as the large red source at the center. The two closest, bright, bluer sources are seen in 2MASS; the brighter of the two is saturated in some of the IRAC bands. The available data for both sources are consistent with the idea that they are heavily reddened background stars, while the colors of L1014-IRS are inconsistent with those of a reddened background star.

The 2MASS point source catalog does not offer measured $J H K$ fluxes for L1014-IRS. However, inspection of the $K$-band quick-look image suggests the presence of faint, extended emission coincident with the central position of L1014-IRS. We have obtained the uncompressed 2MASS image and derived a $K$-band flux, in Table 1 , for an $8^{\prime \prime}$ aperture. This aperture includes all the extended emission that is visible in the 2MASS image.

We observed the $1.2 \mathrm{~mm}$ continuum emission, shown overlain on the $8.0 \mu \mathrm{m}$ data in Figure 2, in 2002 October using the 37 channel MAMBO array (Kreysa et al. 1998) at the IRAM $30 \mathrm{~m}$ telescope in Pico Veleta (Spain). The beam size on the sky was $10^{\prime \prime} 7$. The source was observed on the fly, with the telescope secondary chopping in azimuth by $60^{\prime \prime}$ at a rate of $2 \mathrm{~Hz}$; the total on-target observing time was 85 minutes. Zenith optical depth was 0.2 . The data were reconstructed and analyzed using iterative methods (J. Kauffmann et al. 2004, in preparation). The map was smoothed to $20^{\prime \prime}$. The observed flux for L1014, in Table 1, is calculated within a $120^{\prime \prime}$ aperture centered on the position of a Gaussian fit to the data. The center of the Gaussian is shifted from the IRAC position for L1014-IRS by $\left(-1 . .5,-5{ }^{\prime \prime} 4\right)$.

Finally, we have collected the SCUBA data (850 and $450 \mu \mathrm{m}$ ) of Visser et al. (2002) from the telescope's archive; the $850 \mu \mathrm{m}$ data are shown as contours overlain on the MIPS $24 \mu \mathrm{m}$ image in Figure 2(Plate 2). To provide consistency with our measured flux from MAMBO, we have taken the SCUBA fluxes with a $120^{\prime \prime}$ aperture centered on the position of a Gaussian fit to the data. The 850 and $450 \mu \mathrm{m}$ peaks are shifted from the IRAC position by $\left(-1.1 .5,-8^{\prime \prime}\right)$ and $\left(0^{\prime \prime},-6^{\prime \prime} 9\right)$, respectively. With these continuum data (from $\lambda=3.6-$ $1200 \mu \mathrm{m})$, we calculate $T_{\text {bol }}=50 \mathrm{~K}$ (Myers \& Ladd 1993) and $L_{\mathrm{bol}} / L_{\mathrm{smm}}=20$ (André et al. 1993), classifying L1014IRS as a Class 0 core.

We observed the emission from various molecular transitions with the SRAO $6 \mathrm{~m}$ telescope at Seoul National University (Koo et al. 2003), with a beam size of $\sim 100^{\prime \prime}$. Our CO $(1 \rightarrow 0)$ maps covered $480^{\prime \prime} \times 480^{\prime \prime}$ with a $4^{\prime}$ grid spacing; the ${ }^{13} \mathrm{CO}(1 \rightarrow 0)$ map covered $120^{\prime \prime} \times 120^{\prime \prime}$ on a $2^{\prime}$ grid. We also made single pointing observations toward the center of L1014 in $\mathrm{CS}(2 \rightarrow 1)$ and $\mathrm{HCN}(1 \rightarrow 0)$. The observations of $\mathrm{CO}$ show that there are two different velocity components at about 4 and $-40 \mathrm{~km} \mathrm{~s}^{-1}$ toward L1014 $(\sigma=0.05 \mathrm{~K})$. The $4 \mathrm{~km} \mathrm{~s}^{-1}$ component was detected in CS $(2 \rightarrow 1)\left(T_{A}^{*} \sim 0.22 \mathrm{~K}\right)$ and marginally in $\mathrm{HCN}(1 \rightarrow 0)(0.09 \mathrm{~K})$. The $-40 \mathrm{~km} \mathrm{~s}^{-1}$ component was not detected in either $\mathrm{CS}$ or $\mathrm{HCN}(\sigma=0.03 \mathrm{~K})$.

We also mapped $\mathrm{L} 1014$ in $\mathrm{CO}$ and $\mathrm{C}^{18} \mathrm{O}(1 \rightarrow 0)$ using the SEQUOIA 32 pixel, focal-plane array mounted on the $14 \mathrm{~m}$ FCRAO, with a beam size of $\sim 40^{\prime \prime}$. The spectra were taken in a $800^{\prime \prime} \times 800^{\prime \prime}$ region centered at the $1.2 \mathrm{~mm}$ emission peak in on-the-fly mode. We detected strong emission of $\mathrm{CO}(1 \rightarrow 0)$ $\left(\sigma=0.100 \mathrm{~K} ; \Delta v=0.127 \mathrm{~km} \mathrm{~s}^{-1}\right)$ and $\mathrm{C}^{18} \mathrm{O} \quad(1 \rightarrow 0)$ $\left(\sigma=0.022 \mathrm{~K} ; \Delta v=0.133 \mathrm{~km} \mathrm{~s}^{-1}\right)$ at L1014's velocity $(4.2 \mathrm{~km}$ $\left.\mathrm{s}^{-1}\right)$; this emission extended across our entire map. The spectra are highly non-Gaussian and showed a blue, wing-like emission throughout the map. The $\mathrm{CO}$ emission also has an additional overlapping component redward of the $\mathrm{C}^{18} \mathrm{O}$ line center position $\left(\right.$ at $\left.5 \mathrm{~km} \mathrm{~s}^{-1}\right)$ that peaks at $\left(-200^{\prime \prime},-200^{\prime \prime}\right)$ and is elongated in the southwest-northeast direction. The $\mathrm{C}^{18} \mathrm{O}$ $(1 \rightarrow 0)$ integrated intensity peaks at $\left(-27^{\prime \prime}, 26^{\prime \prime}\right)$ from the reference position; the emission is elongated in the southeastnorthwest direction and is approximately $200^{\prime \prime}$ wide. The component at $v_{\mathrm{LSR}}=-40 \mathrm{~km} \mathrm{~s}^{-1}$ peaks at $\left(32^{\prime \prime},-73^{\prime \prime}\right)$ relative to our reference position. From the FCRAO and SRAO molecular-line observations, we make two conclusions: (1) while the $\mathrm{CO}$ line profiles are non-Guassian, the region is too complicated to either confirm or deny the existence of an outflow, and (2) we see a second velocity component in the $\mathrm{CO}$ and $\mathrm{C}^{18} \mathrm{O}$ spectra at about $-40 \mathrm{~km} \mathrm{~s}^{-1}$.

Brand \& Blitz (1993) assigned a distance of $2.6 \mathrm{kpc}$ to an $\mathrm{H}$ II region, S124, whose associated molecular cloud has $v_{\mathrm{LSR}}=-40 \mathrm{~km} \mathrm{~s}^{-1}$; S124 is offset from L1014-IRS by (3.5, $-0.5)$. This velocity component was not seen in our observations of $\mathrm{HCN}(1 \rightarrow 0)$ and $\mathrm{CS}(2 \rightarrow 1)$. Because no foreground stars are observed within $\sim 1^{\prime}$ of the core's center, the dark cloud as seen in the Digitized Sky Survey (DSS) image cannot be at $2.6 \mathrm{kpc}$, so it must be associated with the $4 \mathrm{~km}$ $\mathrm{s}^{-1}$ component. We believe that the infrared source is also 
associated with the $4 \mathrm{~km} \mathrm{~s}^{-1}$ component, but we consider the alternative possibility.

\section{MODELS FOR THE L1014 CORE}

In an attempt to understand the physical state of L1014, we have used the data to create a working model. We used the SCUBA $850 \mu \mathrm{m}$ data to determine the density profile by matching our models with the observed intensity profile at $850 \mu \mathrm{m}$. We have used a one-dimensional radiative transfer package, DUSTY (Ivezić et al. 1999), to calculate the temperature profile and the observed spectral energy distribution (SED); ObsSphere (Shirley et al. 2002) was used to simulate the SCUBA observations, including the effects of chopping. We used the dust opacities of Ossenkopf \& Henning (1994), which represent grains with thin ice mantles (OH5 dust) and are appropriate for cold, dense cores (e.g., Evans et al. 2001).

The core is heated internally by a protostar and externally by the interstellar radiation field (ISRF). The ISRF has been attenuated with dust whose properties are given by Draine \& Lee (1984) with $A_{V}=0.5$. Both a shallow power-law $\left[n(r) \propto r^{-1.0}\right]$ and a Bonnor-Ebert (BE) sphere were found to provide good fits to the observed $850 \mu \mathrm{m}$ brightness profile, in agreement with results from other "starless" cores (e.g., Evans et al. 2001). Because it offers a good fit to the density structure, we used the analytical approximation given by Tafalla et al. (2002) for an isothermal BE sphere, $n(r)=n_{c} /\left[1+\left(r / r_{0}\right)^{\alpha}\right]$, and found a good fit for $n_{c}=1.5 \times 10^{5} \mathrm{~cm}^{-3}$; for this central density, a BE sphere is approximated with $r_{0}=4090 \mathrm{AU}$ and $\alpha=2.32$. We used inner and outer radii of 50 and 15,000 AU, respectively, but these are poorly constrained. This core has a mass of $1.7 M_{\odot}$.

The long-wavelength dust continuum emission shows that a cold, dense core is present, and external heating can explain the emission longward of $70 \mu \mathrm{m}$. The bolometric luminosity for L1014-IRS is $0.3 L_{\odot}$, typical of starless cores heated only by the ISRF (Evans et al. 2001). In these respects, L1014 looks like a core with no significant internal heating.

The IRAC and MIPS data trace the SED of a considerably warmer source. The starless core model underestimates the $70 \mu \mathrm{m}$ flux by a factor of 50 and cannot begin to explain the data at shorter wavelengths. Given the parameters above, we find that a source with luminosity of $0.025 L_{\odot}$ and $T_{\text {eff }}=$ $700 \pm 300 \mathrm{~K}$ best fits the IRAC data (as seen in Fig. 3).

To match the 24 and $70 \mu \mathrm{m}$ points, a cooler component with substantially more surface area, such as a disk, is required. We use a simple model (Butner et al. 1994; Adams et al. 1988) to simulate the disk emission. The disk has a total mass (gas and dust) of $4 \times 10^{-4}\left(R_{D} / 50 \mathrm{AU}\right)^{0.5} M_{\odot}$ and a temperature profile, $T(r) \propto r^{-0.5}$, simulating the effects of accretion onto the disk surface. The outer radius of the disk, $R_{D}$, is set to $50 \mathrm{AU}$, the smallest disk that matches the $70 \mu \mathrm{m}$ data; larger values for $R_{D}$ and the mass are possible.

The disk model that fits is passively heated by the central protostar and is itself luminous, possibly from accretion directly onto the disk. The partition of luminosity between the star and disk is not well constrained, so we use $0.09 L_{\odot}$ for the total luminosity of a central source in further discussion. Future observations with millimeter and submillimeter interferometers are needed to constrain the value of $R_{D}$ and hence the mass; this model, with $R_{D}=50 \mathrm{AU}$, predicts a flux of $4.3 \mathrm{mJy}$ at $850 \mu \mathrm{m}$.

If this model is correct, we are observing a very low mass protostar (or proto-brown dwarf). Assuming spherical accretion at a rate of $2 \times 10^{-6} M_{\odot} \mathrm{yr}^{-1}$ (as expected for a $10 \mathrm{~K}$,

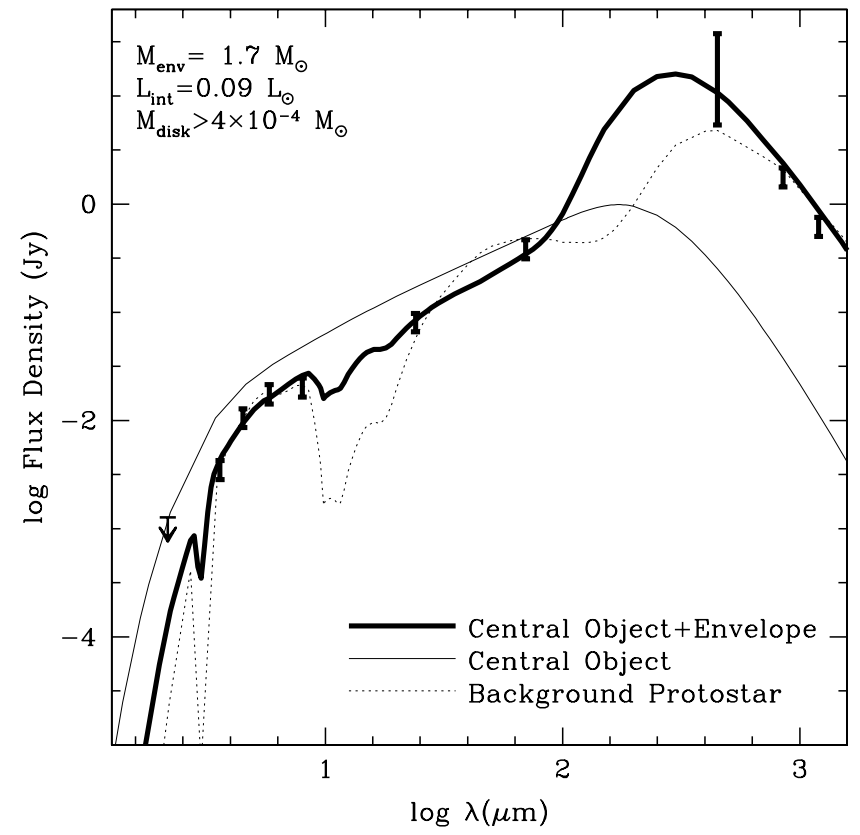

FIG. 3.-Model of the SED for L1014-IRS, assuming a distance of $200 \mathrm{pc}$. This model shows the emission from a BE sphere heated externally by the ISRF and internally by a star and disk. The star has a luminosity of $0.025 L$. and an effective temperature of $700 \mathrm{~K}$. The disk is made to simulate flaring, has a mass of $4 \times 10^{-4} M_{\odot}$, and has an intrinsic luminosity of $0.065 L_{\odot}$. The spectrum for the internal source is drawn by the thin, solid line; this is the input spectrum for DUSTY. The emergent SED is represented with the thicker line. The thin, dotted line shows the model for a background protostar, as discussed in the text.

collapsing, isothermal sphere) onto a star with a radius of $3 R_{\odot}$ and a mass of $0.1 M_{\odot}$, the luminosity would be $1.8 L_{\odot}$, 20 times what the observations permit. If we consider accretion rates appropriate for the BE or "Plummer-like" sphere (e.g., $3 \times 10^{-5} M_{\odot} \mathrm{yr}^{-1}$ ), as in Whitworth \& Ward-Thompson (2001), the disparity between the predicted and observed luminosity is greatly amplified. Kenyon \& Hartmann (1995) suggested that protostars may appear subluminous if accretion occurs toward the edge of the circumstellar disk. As the disk becomes too massive, the system will undergo an FU Oritype event in which material accretes onto the star in a short burst. Perhaps we see L1014-IRS during a quiescent time, when material is accreting and collecting in the disk. Nonetheless, this is not an ordinary accreting pre-main-sequence star. Unless accretion is much less or the radius much larger, the mass of the central object is possibly substellar.

These conclusions depend on the distance, which is uncertain. Roughly, the core mass and central luminosity scale as $d^{2}$. For $400 \mathrm{pc}$, the values would be $6.8 \mathrm{M}_{\odot}$ and $0.36 L_{\odot}$. Furthermore, the geometry might vary from the one-dimensional core model studied in this paper. We have created a simple twodimensional model that includes a completely evacuated outflow cavity with a $30^{\circ}$ opening angle and an internal source as already described. Using RADMC (Dullemond \& Dominik 2004), we have calculated the SED for this model and found that, unless you look into the outflow cavity, the SED does not change by more than $10 \%$.

Another possible alternative is that L1014-IRS is a background protostar in the $-40 \mathrm{~km} \mathrm{~s}^{-1}$ cloud at $2.6 \mathrm{kpc}$. Models were calculated for an embedded protostar at this distance obscured by a foreground cloud; the emergent SED for this model is shown in Figure 3. This was calculated in two steps in DUSTY, first calculating the emerging spectrum of the 
protostar and then illuminating a $10 \mathrm{~K}$ planar slab of $A_{V}=10$ with this spectrum. The protostellar envelope was assumed to be free falling $\left[n=n_{0}\left(r / r_{0}\right)^{-3 / 2}\right]$, and the ISRF was ignored. The modeled protostar had $L=16 L_{\odot}$ and was surrounded by a $0.6 M_{\odot}$ envelope but no disk. The infrared source could be a typical protostar at a larger distance. We find this scenario to be statistically unlikely for several reasons. First, the lack of $\mathrm{CS}$ and $\mathrm{HCN}$ emission from the $-40 \mathrm{~km} \mathrm{~s}^{-1}$ component and the fact that the $\mathrm{CO}$ and ${ }^{13} \mathrm{CO}$ emission do not peak at this position argue against this scenario. Second, a map of CS $(2 \rightarrow 1)$ at FCRAO with a $50^{\prime \prime}$ beam shows that the $4 \mathrm{~km} \mathrm{~s}^{-1}$ component has peak emission within one beam of L1014-IRS, further supporting a nearby distance (A. Crapsi et al. 2004, in preparation). Finally, at $70 \mu \mathrm{m}, \mathrm{L} 1014-\mathrm{IRS}$ was the only source of emission within our field; the $24 \mu \mathrm{m}$ flux for this source was the largest within the MIPS field for L1014. Such bright infrared objects are uncommon, so a chance alignment between a background protostar and a foreground starless core is unlikely.

\section{CONCLUSIONS}

We have presented one of the most comprehensive sets of continuum data for a "starless" core since their discovery. These data span wavelengths from 2.17 to $1200 \mu \mathrm{m}$ and include spatially resolved maps of the millimeter emission from L1014-IRS. From these data, we conclude that this core, previously thought to be starless, does harbor a central object. Assuming a distance of $200 \mathrm{pc}$, we have created a model for this object that includes a $1.7 M_{\odot}$ envelope heated externally by the ISRF. This core contains a central object with low luminosity $\left(\sim 0.09 L_{\odot}\right)$. This central source must have stellar and disklike components in order to match the 3.6-70 $\mu \mathrm{m}$ photometry. This object appears to be substellar, although there is enough envelope mass to grow a star if a substantial fraction accretes. We have considered an alternate scenario, in which a distant, more luminous but less embedded protostar lies behind the core of the L1014 dark cloud. This scenario is unlikely but cannot be ruled out conclusively without further observations.

Assuming that the infrared source lies in the L1014 cloud, there are two significant conclusions. First, cores thought to be starless may harbor central objects. If this phenomenon is common, estimates for timescales for the evolution of starless cores will need revision. Second, for plausible distances, this source has a very low luminosity. The usual assumptions about accretion rates and pre-main-sequence masses and radii produce far more luminosity than is seen. This object currently appears to be substellar, although it is likely to accrete more mass.

Support for this work, part of the Spitzer Legacy Science Program, was provided by NASA through contracts 1224608 and 1230780 issued by the Jet Propulsion Laboratory, California Institute of Technology, under NASA contract 1407. The Leiden c2d legacy team is supported by a Spinoza grant from the Netherlands Foundation for Scientific Research and by a grant from the Netherlands Research School for Astronomy. Observations at the SRAO were supported by the KOSEF R01-2003-000-10513-0 program. The SCUBA data were provided to us as a guest user at the Canadian Astronomy Data Centre, which is operated by the Dominion Astrophysical Observatory for the National Research Council of Canada's Herzberg Institute of Astrophysics. This publication makes use of data products from 2MASS, which is a joint project of the University of Massachusetts and the Infrared Processing and Analysis Center, California Institute of Technology, funded by NASA and the NSF. Finally, we thank our anonymous referee for insightful and useful comments.
Adams, F. C., Shu, F. H., \& Lada, C. J. 1988, ApJ, 326, 865

Allen, L. E., et al. 2004, ApJS, 154, 363

André, P., Ward-Thompson, D., \& Barsony, M. 1993, ApJ, 406, 122

Brand, J., \& Blitz, L. 1993, A\&A, 275, 67

Butner, H. M., Natta, A., \& Evans, N. J., II. 1994, ApJ, 420, 326

Draine, B. T., \& Lee, H. M. 1984, ApJ, 285, 89

Dullemond, C. P., \& Dominik, C. 2004, A\&A, 417, 159

Evans, N. J., II, Rawlings, J. M. C., Shirley, Y. L., \& Mundy, L. G. 2001, ApJ, 557,193

Evans, N. J., II, et al. 2003, PASP, 115, 965

Fazio, G. G., et al. 2004, ApJS, 154, 10

Ivezić, Ž., Nenkova, M., \& Elitzur, M. 1999, User Manual For DUSTY (Lexington: Univ. Kentucky)

Kenyon, S. J., \& Hartmann, L. W. 1995, ApJS, 101, 117

Koo, B.-C., et al. 2003, J. Korean Astron. Soc., 36, 43

\section{REFERENCES}

Kreysa, E., et al. 1998, Proc. SPIE, 3357, 319

Lynds, B. T. 1962, ApJS, 7, 1

Myers, P. C., \& Benson, P. J. 1983, ApJ, 266, 309

Myers, P. C., \& Ladd, E. F. 1993, ApJ, 413, L47

Ossenkopf, V., \& Henning, T. 1994, A\&A, 291, 943

Rieke, G. H., et al. 2004, ApJS, 154, 25

Schechter, P. L., Mateo, M., \& Saha, A. 1993, PASP, 105, 1342

Shirley, Y. L., Evans, N. J., II, \& Rawlings, J. M. C. 2002, ApJ, 575, 337

Tafalla, M., Myers, P. C., Caselli, P., Walmsley, C. M., \& Comito, C. 2002, ApJ, 569, 815

Visser, A. E., Richer, J. S., \& Chandler, C. J. 2002, AJ, 124, 2756

Ward-Thompson, D., André, P., \& Kirk, J. M. 2002, MNRAS, 329, 257

Werner, M., et al. 2004, ApJS, 154, 1

Whitney, B. A., Wood, K., Bjorkman, J. E., \& Cohen, M. 2003, ApJ, 598, 1079

Whitworth, A. P., \& Ward-Thompson, D. 2001, ApJ, 547, 317 


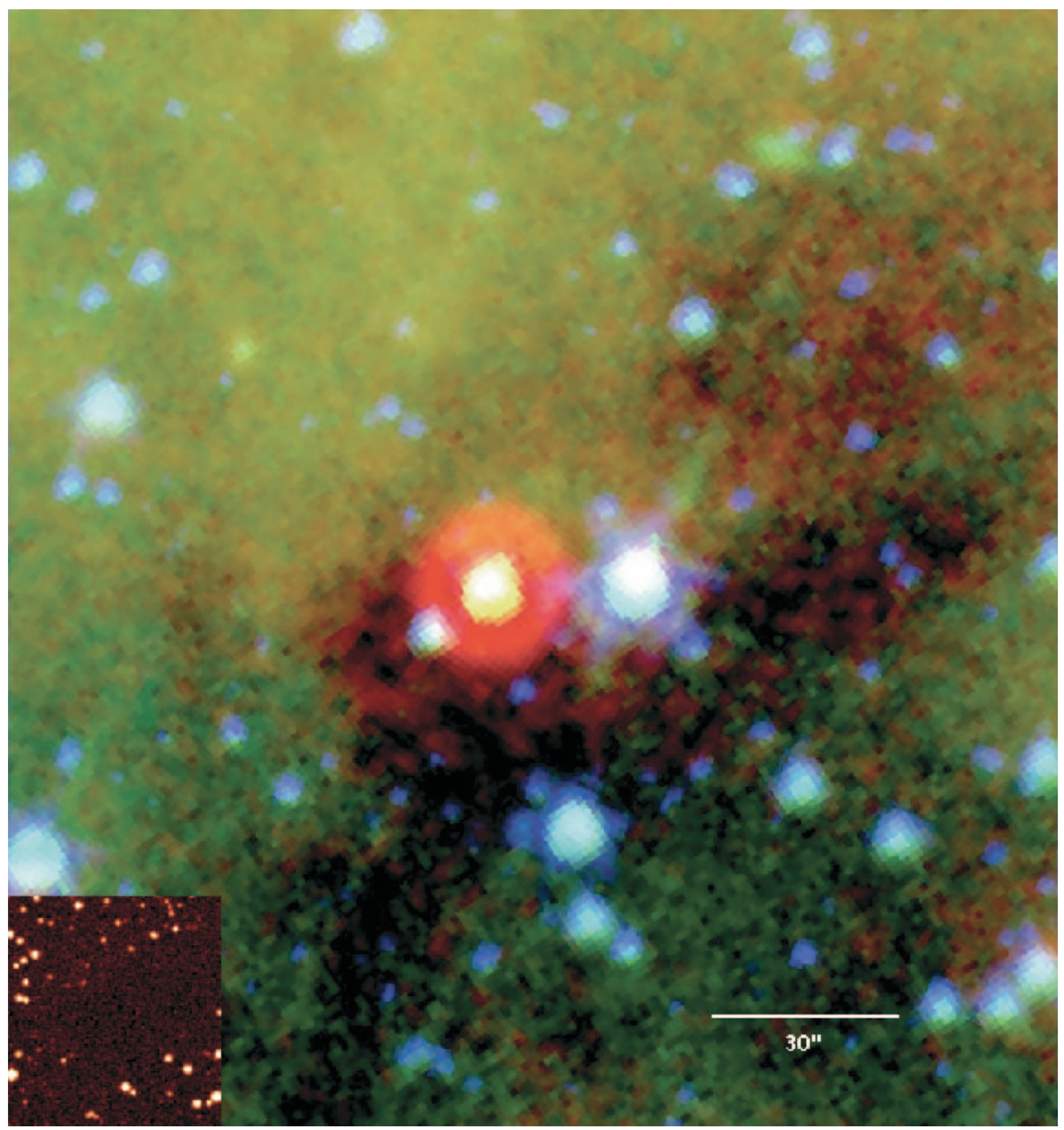

FIG. 1.-Three-color composite image of L1014-IRS. The IRAC 3.6 and $8.0 \mu \mathrm{m}$ data are represented here as blue and green, while the MIPS $24 \mu \mathrm{m}$ data, with resolution more than 3 times worse than that of the IRAC bands, are shown in red. The $R$-band image, at lower left, is of the same field as DSS-2. The scale and orientation are the same as in Fig. 2. 


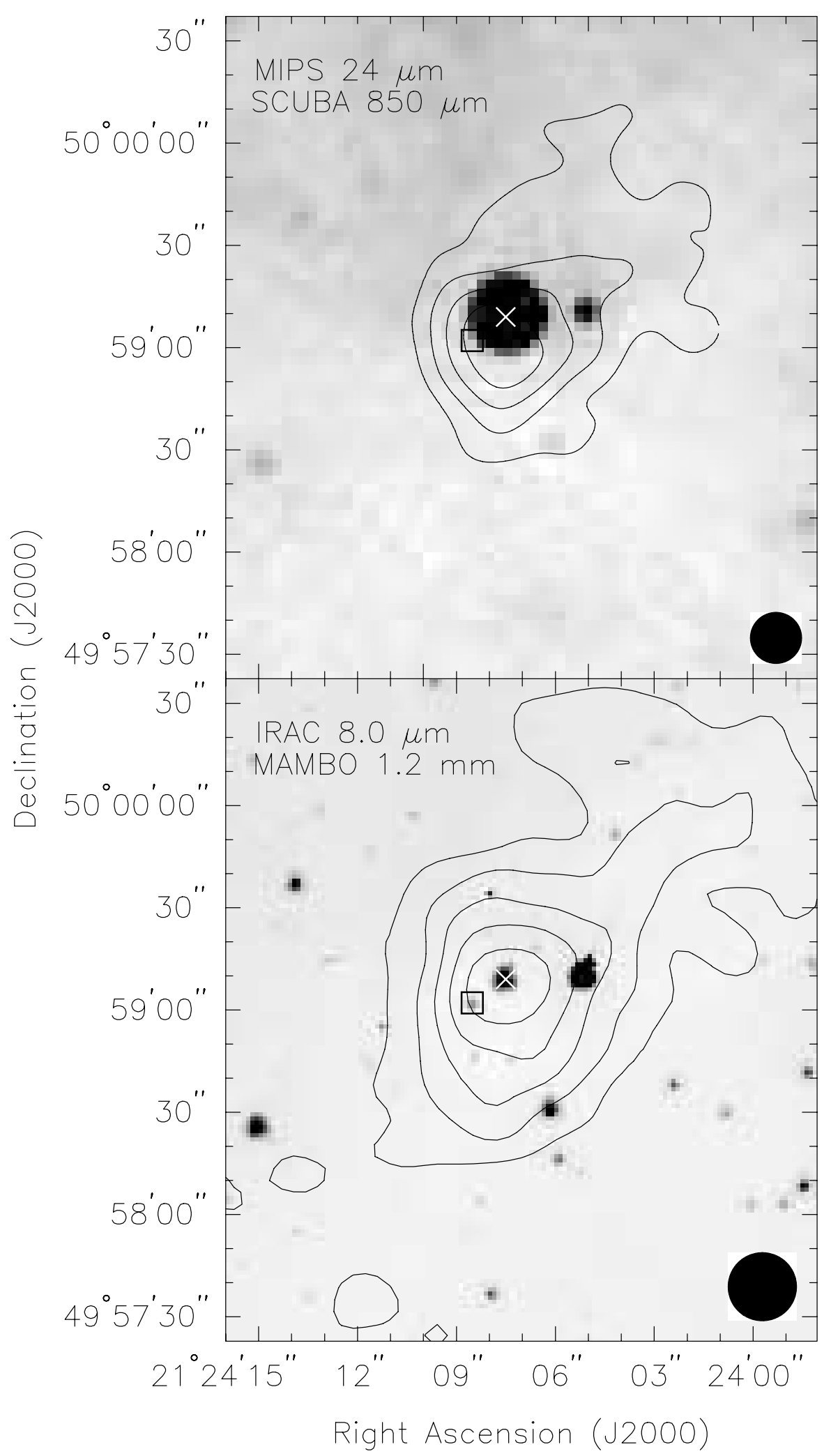

FIG. 2.-Bottom: The $8.0 \mu \mathrm{m}$ data from IRAC in gray scale, with the MAMBO $1.2 \mathrm{~mm}$ data as contours. The contours begin at $3 \sigma\left(3 \sigma=3.9 \mathrm{mJy}^{\circ}\right.$ beam $\left.{ }^{-1}\right)$ and increase by $3 \sigma$. The white cross marks the position of L1014-IRS $\left(\alpha=21^{\mathrm{h}} 24^{\mathrm{m}} 07^{\mathrm{s}} .51, \delta=+49^{\circ} 59^{\prime} 09^{\prime \prime} .0[\mathrm{~J} 2000.0]\right)$. The data have been smoothed by $20^{\prime \prime}$, as shown by the black circle. Top: The $24 \mu \mathrm{m}$ data from MIPS in gray scale, with the SCUBA $850 \mu \mathrm{m}$ data as contours. The contours begin at $3 \sigma$ and increase by $3 \sigma$. The black circle shows the resolution of the James Clerk Maxwell Telescope at $850 \mu \mathrm{m}, 15^{\prime \prime}$. The white cross marks the position of L1014-IRS $\left(\alpha=21^{\mathrm{h}} 24^{\mathrm{m}} 07.51\right.$, $\delta=+49^{\circ} 59^{\prime} 09^{\prime \prime} .0$ [J2000.0]). In addition, 2MASS 21240852+4959021, as discussed in the text, is marked by a white square. 